\title{
Eccrine Angiomatous Hamartoma
}

National Cancer Institute

\section{Source}

National Cancer Institute. Eccrine Angiomatous Hamartoma. NCI Thesaurus. Code C4703.

A rare, congenital skin lesion consisting of a proliferation of eccrine glands with a vascular stroma. It presents as a flesh-colored, hyperhidrotic, painful papule or plaque appearing at birth or during childhood. 